\title{
Ten years' experience with intraoperative MRI-assisted transsphenoidal pituitary surgery
}

\author{
Michal Hlaváč, MD, ${ }^{1}$ Andreas Knoll, MD, ${ }^{1}$ Benjamin Mayer, PhD, ${ }^{3}$ Michael Braun, MD, ${ }^{4}$ \\ Georg Karpel-Massler, MD, PhD, ${ }^{1}$ Gwendolin Etzrodt-Walter, MD, ${ }^{2}$ Jan Coburger, MD, PhD, ${ }^{1}$ \\ Christian Rainer Wirtz, MD, PhD, ${ }^{1}$ and Andrej Pal'a, MD'
}

${ }^{1}$ Department of Neurosurgery, University of Ulm, Günzburg; ${ }^{2}$ Endokrinologiezentrum Ulm; ${ }^{3}$ Institute of Epidemiology and Medical

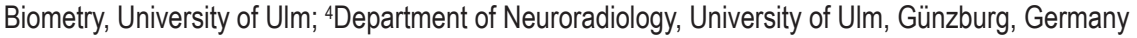

OBJECTIVE Many innovations have been introduced into pituitary surgery in the quest to maximize the extent of tumor resection. Because of the deep and narrow surgical corridor as well as the heterogeneity of confronted pathologies, anatomical orientation and identification of the target tissue can become difficult. Intraoperative MRI (iMRI) may have the potential to increase extent of resection (EOR) in transsphenoidal pituitary surgery. Furthermore, it may simplify anatomical orientation and risk assessment in difficult cases. Here, the authors evaluated the additional value of iMRI for the resection of pituitary adenomas performed in the past 10 years in their department.

METHODS They performed a retrospective single-center analysis of patients treated for pituitary adenoma in their department after the introduction of iMRI between 2008 and 2018. Of 495 transsphenoidal approaches, 300 consecutive MRIassisted surgeries for pituitary adenomas encompassing 294 patients were selected for further analysis. Microscopic, endoscopic, or endoscope-assisted microscopic transsphenoidal approaches were distinguished. EOR as well as additional resection following iMRI was evaluated via detailed volumetric analysis. Patients were stratified according to the Knosp adenoma classification. Furthermore, demographic data, clinical symptoms, endocrine outcome, and complications were evaluated. Univariable and multivariable Cox regression analyses of progression-free survival (PFS) were performed.

RESULTS Pituitary adenomas classified as Knosp grades $0-2$ were found in $60.3 \%$ of cases ( $n=181)$. The most common tumors were nonfunctioning adenomas (75\%). Continued resection following iMRI significantly increased EOR $(7.5 \%, p<0.001)$ and the proportion of gross-total resections (GTRs) in transsphenoidal pituitary surgery $(54 \%$ vs $68.3 \%$, $p<0.001)$. Additional resection after iMRI was performed in $37 \%$ of cases. Only in the subgroup of patients with Knosp grades $0-2$ adenomas treated with the microsurgical technique was additional resection significantly more common than in the endoscopic group $(p=0.039)$. Residual tumor volume, Knosp grade, and age were confirmed as independent predictors of PFS ( $p<0.001, p=0.021$, and $p=0.029$, respectively) in a multivariable Cox regression analysis. Improvement of visual field deficits was documented in $78.6 \%$ of patients whose optic apparatus had been affected preoperatively. Revision surgery was done in $7.3 \%$ of cases; in $5.6 \%$ of cases, it was performed for cerebrospinal fluid fistula.

CONCLUSIONS In this series, iMRI led to the detection of a resectable tumor remnant in a high proportion of patients, resulting in a greater EOR and higher proportion of GTRs after continued resection in microsurgical and endoscopic transsphenoidal resection of pituitary adenomas. The volume of residual tumor was the most important predictor of PFS. Given the study data, the authors postulated that every bit of removed tumor serves the patient and increases their chances of a favorable outcome.

https://thejns.org/doi/abs/10.3171/2020.3.FOCUS2072

KEYWORDS transsphenoidal pituitary surgery; intraoperative MRI; extent of resection; progression-free survival; endoscopic technique; microscopic technique

$\mathrm{S}$ INCE the introduction of intraoperative MRI (iMRI) in pituitary surgery, the method has experienced divided acceptance. So far, no prospective randomized trial has demonstrated a clear benefit in terms of extent of resection (EOR) and the rate of gross-total re- section (GTR). Even though this level of evidence will be difficult to achieve, a growing number of publications have supported a positive influence of EOR on progression-free survival (PFS). ${ }^{4,20}$ With the introduction of endoscopy to transsphenoidal pituitary surgery, the interest in iMRI

ABBREVIATIONS CSF = cerebrospinal fluid; $\mathrm{DI}=$ diabetes insipidus; EOR = extent of resection; $\mathrm{GH}=$ growth hormone; $\mathrm{GTR}=$ gross-total resection; iMRI = intraoperative MRI; PFS = progression-free survival; ROC = receiver operating characteristic.

SUBMITTED January 31, 2020. ACCEPTED March 3, 2020

INCLUDE WHEN CITING DOI: 10.3171/2020.3.FOCUS2072. 
seems to have waned. Even so, a recent publication has suggested a synergistic effect of endoscopy and iMRI on EOR. ${ }^{19,20}$ Direct comparisons of microscopic and endoscopic surgical techniques have thus far failed to establish the superiority of either technique. ${ }^{6,11,17,22}$ We hypothesize that iMRI allows one to identify occult tumor remnants not recognized by either microscopy or endoscopy. With the help of navigation, iMRI data can precisely guide resection of these remnants. Thus, the EOR will remain limited solely by the surgical corridor and infiltration of critical anatomical structures. Extended endoscopic approaches could allow resection of tumor beyond the reach of the standard microscopic approach.

In order to test this hypothesis, we performed a retrospective institutional review of all iMRI cases of transsphenoidal pituitary adenoma resection. We have evaluated the influence of iMRI on EOR in the setting of different surgical techniques. Additionally, we analyzed endocrine outcome and performed a subgroup analysis of patients with adenomas classified as Knosp grades 0-2, since one might expect these tumors to be amenable to complete resection without additional intraoperative imaging.

\section{Methods}

\section{Patients and Follow-Up}

Beginning with complex cases after implementation, iMRI-guided pituitary surgery soon became the standard of treatment for all pituitary adenomas at our institution. All surgeries between 2008 and 2018 in which the transsphenoidal approach had been performed were assessed retrospectively $(n=495)$. Patients with a diagnosis other than pituitary adenoma were excluded $(\mathrm{n}=182)$. Of the remaining 313 patients, 13 were treated without iMRI because of contraindications to MRI, an emergent surgical procedure, or issues with the availability of iMRI. All consecutive patients with a final histopathological diagnosis of pituitary adenoma and who had undergone iMRIassisted transsphenoidal resection were included in our study.

Pituitary adenomas were graded according to criteria published by Knosp et al., ${ }^{9}$.13 For subgroup analysis, less invasive adenomas were defined as Knosp grades 0-2 and invasive adenomas as Knosp grades 3-4. Postoperative assessment of clinical and endocrine status as well as MRI was performed 3-12 months after surgery. Thereafter, serial MRI was performed on a yearly basis. PFS was defined as new tumor suspected on follow-up MRI or an increase of postoperative tumor remnant in the case of subtotal resection. In hormone-producing tumors, recurrence of hormone excess was defined as progression. Basic demographic data (age, sex) as well as basic adenoma characteristics (recurrent adenoma, subtype of adenoma, apoplectic adenoma) were recorded.

Pituitary function was evaluated in cooperation with endocrinologists in a multidisciplinary manner before surgery, 4-6 weeks after surgery, and 3-6 months after surgery. The endocrine evaluation included baseline pituitary hormone levels and hypoglycemic testing for determination of cortisol and growth hormone $(\mathrm{GH})$ dynamics.
Where these tests were contraindicated, a corticotropinreleasing hormone stimulation test was performed instead. New hypopituitarism was defined as worsening of endocrine function of one or more pituitary axes after surgery. New permanent diabetes insipidus (DI) was recorded as an additional parameter in our analysis.

Disease remission in patients with acromegaly was defined as a normal insulin-like growth factor 1 (IGF-1) level and either GH suppressed to $<0.4 \mathrm{ng} / \mathrm{ml}$ during an oral glucose tolerance test or $\mathrm{GH}$ level $<1.0 \mathrm{ng} / \mathrm{ml}$ on random sampling. Remission in Cushing's disease had occurred either if cortisol substitution was necessary or if the morning cortisol level was within normal range with positive suppression to below $2 \mu \mathrm{g} / \mathrm{dl}$ after low-dose dexamethasone exposure.

Furthermore, visual deficits due to tumor-induced compression of the optic apparatus were documented before surgery. Visual field testing was repeated regularly after surgery. Improvement, stable state, and worsening of visual function were evaluated.

\section{Operating Room Setup and MRI}

An intraoperative 1.5-T MRI scanner (Espree, Siemens AG) has been available as a one-room solution at our department since October 2008. The analysis of intraoperative residual tumor was performed on thin-slice $(2 \mathrm{~mm})$, high-resolution, coronal and sagittal T2-weighted and contrast-enhanced T1-weighted images using Brainlab Elements software (Brainlab AG). Postoperative MRI was performed 3 months after surgery. Pre- and postoperative MR images were acquired with either the intraoperative scanner or the Symphony 1.5-T MRI system (Siemens AG). iMRI with all mentioned sequences generally led to an additional surgical time of 45 minutes.

\section{MRI Volumetric Assessment}

Preoperative MR images included coronal T2-weighted turbo spin echo as well as coronal and sagittal T1weighted plain and contrast-enhanced sequences. Tumor volume was measured after image fusion using the abovementioned software. Tumor borders were segmented semi-automatically on coronal and sagittal T2-weighted and gadolinium-enhanced T1-weighted images. For the volumetric resection analysis, we used preoperative, intraoperative, and postoperative MRI data, the latter acquired 3 months after surgery. If tumor borders were ambiguous, additional MR images 1 year after surgery were used for the evaluation. GTR was presumed if postoperative MRI showed no suspicious remnants. EOR was calculated as the volumetric proportion of tumor volume remnant as compared to the preoperative tumor volume. We calculated the EOR based on the iMRI and the postoperative MRI 3 months after surgery. MRI assessments and volumetric analysis were performed in cooperation with the Department of Neuroradiology. All MRI scans were reviewed by a radiologist and experienced neurosurgeon. An experienced radiologist is always available for the evaluation of iMRI. Additionally, intraoperative imaging offers high image quality, and artifacts that could influence decisionmaking are rare. 
TABLE 1. Patients and tumor characteristics

\begin{tabular}{lcccc}
\hline \multicolumn{1}{c}{ Parameter } & Total & Microsurgery Cohort & Endoscopy Cohort & Microsurgical Endoscope-Assisted Cohort \\
\hline No. of surgeries (\%) & 300 & $187(62.3)$ & $100(33.3)$ & $13(4.3)$ \\
\hline Mean age in yrs (SD) & 55 & $56(15)$ & $54(16)$ & $54(15)$ \\
\hline No. of male cases (\%) & $193(64.3)$ & $125(66.8)$ & $61(61)$ & $7(53.8)$ \\
\hline Mean tumor vol in cm ${ }^{3}(\mathrm{SD})$ & $7.4(7.9)$ & $6.9(7.1)$ & $8.2(9.4)$ & $8.2(5.9)$ \\
\hline Knosp grade, no. of cases (\%) & & & & $10(76.9)$ \\
\hline $0-2$ & $181(60.3)$ & $121(64.7)$ & $50(50)$ & $3(23.1)$ \\
\hline 3-4 & $119(39.7)$ & $66(35.3)$ & $50(50)$ & $11(84.6)$ \\
\hline Adenoma subtype, no. of cases & & & & $1(7.7)$ \\
(\%) & & & $72(72)$ & - \\
\hline Nonfunctioning & $225(75)$ & $142(75.9)$ & $17(17)$ & - \\
\hline GH & $44(14.7)$ & $26(13.9)$ & $9(9)$ & - \\
\hline ACTH & $20(6.7)$ & $11(5.9)$ & $2(2)$ & - \\
\hline Prolactinoma & $7(2.3)$ & $5(2.7)$ & - & $1(7.7)$ \\
\hline TSH & $1(0.3)$ & $1(0.5)$ & $15(15)$ & $2(15.4)$ \\
\hline FSH & $3(1)$ & $2(1.1)$ & $5(5)$ & - \\
\hline Recurrent adenoma & $50(16.7)$ & $33(17.6)$ & $11(5.9)$ & \\
\hline Apoplectic adenoma & $16(5.3)$ & & & \\
\hline ACTH & & & \\
\hline
\end{tabular}

$\mathrm{ACTH}=$ adrenocorticotropic hormone; $\mathrm{FSH}=$ follicle-stimulating hormone; $\mathrm{TSH}=$ thyroid-stimulating hormone.

\section{Surgical Procedure}

The endoscopic transsphenoidal approach has been performed by two neurosurgeons in our department since 2015. Rigid $0^{\circ}, 30^{\circ}$, and $45^{\circ}$ Hopkins endoscopes with the four-hands technique were used intraoperatively. In some cases of invasive adenomas with skull base infiltration, extended endoscopic approaches were performed in cooperation with ear, nose, and throat surgeons. The microscopic approach was performed by four experienced neurosurgeons using a direct unilateral transnasal paraseptal route. A navigation system (Brainlab AG) was used in both the endoscopic and microscopic procedures.

Skull base reconstruction was performed with a fibrincoated sponge in cases of small or no intraoperative cerebrospinal fluid (CSF) leaks. Large defects were sealed using a multilayer technique with an abdominal subcutaneous fat graft and fibrin-coated sponge or nasoseptal flap, according to our internal protocol.

\section{Surgical Complications}

Postoperative meningitis was assumed when antibiotic treatment was initiated because of typical clinical signs of meningeal inflammation, even if no pathogen was isolated. A CSF fistula was considered a complication if a lumbar drain unrelated to the surgical procedure or revision surgery was necessary. Furthermore, intra- and postoperative bleeding, thromboembolic complications, and permanent or transient new neurological deficits were included. Hypopituitarism and DI were evaluated separately as described above.

\section{Data Analysis}

Statistical analysis was performed using SPSS 26.0 (IBM Corp.). Descriptive statistics as well as the Mann-
Whitney U-test and Fisher exact test were used for the data analysis. Univariable and multivariable Cox regression models for PFS were calculated. Influencing variables were age, initial tumor volume, sex, Knosp grade, recurrent surgery, EOR, and adenoma subtype. We did not include surgical technique in this analysis since endoscopy was routinely used after 2015 . All variables achieving significance $(\mathrm{p}<0.05)$ in the univariable analysis were included in the multivariable analysis.

The study was conducted in accordance with the Declaration of Helsinki. The approval of the local ethics committee of Ulm University was obtained.

\section{Results \\ General Characteristics}

During the study period, 300 adenomectomies were performed in 294 patients with iMRI and were included in this analysis. Male patients were almost twice as common as female patients. Nonfunctioning adenomas were found in three-quarters of the cases. Recurrent adenomas were treated in $16.7 \%$ of cases. Apoplexy as an initial syndrome was relatively rare. An overview of the techniques used during surgery together with basic characteristics for each group is provided in Table 1. The mean follow-up was 31 months (95\% CI 28-34 months), and the median follow-up was 24 months.

\section{Extent of Resection and iMRI}

According to the volumetric analysis, additional resection after iMRI significantly increased the final EOR $(7.5 \%, \mathrm{p}<0.001$; Table 2$)$. This difference remained significant even if microsurgical and endoscopic cohorts were examined separately $(\mathrm{p}<0.001$; Table 2$)$. This fact is underlined by the relatively high frequency of additional 
TABLE 2. GTR rates and endocrinological results

\begin{tabular}{lcccc}
\hline \multicolumn{1}{c}{ Parameter } & Total & Microsurgical Cohort & Endoscopic Cohort & $p$ Value \\
\hline Mean intraop vol in $\mathrm{cm}^{3}(\mathrm{SD})$ & $1.029(3.03)$ & $1.068(3.17)$ & $1.07(2.98)$ & 0.835 \\
\hline Mean postop vol in $\mathrm{cm}^{3}(\mathrm{SD})$ & $0.472(2.36)$ & $0.500(2.08)$ & $0.490(2.97)$ & 0.296 \\
\hline p value & $<0.001$ & $<0.001$ & $<0.001$ & \\
\hline Intraop GTR (\%) & $162 / 300(54)$ & $96 / 187(51.3)$ & $57 / 100(57)$ & 0.360 \\
\hline Postop GTR (\%) & $200 / 293(68.3)$ & $119 / 187(63.6)$ & $68 / 100(68)$ & 0.300 \\
\hline p value & $<0.001$ & $<0.001$ & $<0.001$ & \\
\hline $\begin{array}{l}\text { Additional resection after iMRI } \\
(\%)\end{array}$ & $111 / 300(37)$ & $73 / 187(39)$ & $34 / 100(34)$ & 0.401 \\
\hline $\begin{array}{l}\text { New hypopituitarism after surgery } \\
(\%)\end{array}$ & $66 / 234(28.2)$ & $49 / 165(29.7)$ & $14 / 80(17.5)$ & 0.041 \\
\hline
\end{tabular}

Boldface type indicates statistical significance.

resections. We found no significant difference in the rate of additional resections between the endoscopic and microscopic techniques.

Comparing GTR on the iMRI and the postoperative MRI scan, the rate of GTR in the postoperative scan after additional resection was significantly increased (Table 2). No significant difference was found in the volume of tumor remnants or GTR rates between microscopic and endoscopic cohorts.

In $11.3 \%(n=34)$ of the procedures, visible tumor remnant was found on iMRI, but no further resection was performed. Despite this fact, we found a significant decrease in tumor volume between the iMRI and the 3-month follow-up MRI ( $\mathrm{p}<0.001)$ in these patients.

\section{Knosp Grade 0-2 Pituitary Adenomas}

In the subgroup analysis of less invasive pituitary adenomas classified as Knosp grades 0-2, continued resection after iMRI resulted in significantly lower postoperative tumor volume and higher rate of GTR (Table 3). This significance persisted for postoperative tumor volume even if microscopic or endoscopic approaches were evaluated separately. GTR rates after iMRI remained significantly increased in the microscopic cohort but failed to reach significance in the endoscopic cohort. The number of additional resections was significantly higher in the patients treated via the microscopic approach. Nevertheless, $16 \%$ of the patients treated by the endoscopic technique benefited from additional resection of tumor remnant detected by iMRI. Interestingly, we found a significant difference in favor of the endoscopic technique with regard to the final EOR and proportion of GTRs. Furthermore, additional resection was significantly more common with the microscopic technique.

\section{Progression-Free Survival}

In terms of longer PFS, age, GTR, Knosp grade, and initial tumor volume achieved a significant difference in the univariable Cox regression analysis. In the multivariable model, only GTR was confirmed as an independent prognostic variable for longer PFS (Fig. 1 and Table 4). The mean PFS was 84 months (95\% CI 78-90 months). The median PFS of patients after STR was 62 months (95\% CI 49.616-74.384 months). The median PFS of patients after GTR could not be estimated since adenoma recurrence was rare in this cohort. The log-rank test confirmed a significant difference between the GTR and STR groups ( $\mathrm{p}<0.001)$. Surgical technique was not included in the analysis since endoscopy has been routinely used after 2015. Additionally, we created a second model in which residual tumor volume was substituted for GTR. In addition to residual tumor volume, age and Knosp grade became significant in terms of prolonged PFS in the multivariable Cox regression analysis (Table 5). Furthermore, a receiver operating characteristic (ROC) curve model was calculated in order to define the cutoff value for residual

TABLE 3. EOR and endocrinological results in Knosp grade $0-2$ adenomas

\begin{tabular}{lcccc}
\hline \multicolumn{1}{c}{ Parameter } & Total & Microsurgical Cohort & Endoscopic Cohort & $p$ Value \\
\hline Mean intraop vol in $\mathrm{cm}^{3}(\mathrm{SD})$ & $0.270(1.05)$ & $0.325(1.15)$ & $0.158(0.88)$ & $\mathbf{0 . 0 4 4}$ \\
\hline Mean postop vol in $\mathrm{cm}^{3}(\mathrm{SD})$ & $0.062(0.63)$ & $0.095(0.78)$ & $0.0005(0.003)$ & $\mathbf{0 . 0 2 4}$ \\
\hline p value & $<0.001$ & $<0.001$ & $\mathbf{0 . 0 4 3}$ & \\
\hline Intraop GTR (\%) & $130 / 181(71.8)$ & $81 / 121(66.9)$ & $41 / 50(82)$ & $\mathbf{0 . 0 4 8}$ \\
\hline Postop GTR (\%) & $154 / 181(85.1)$ & $97 / 118(82.2)$ & $47 / 48(97.9)$ & $\mathbf{0 . 0 0 7}$ \\
\hline p value & $<0.001$ & $<0.001$ & 0.167 & 0.039 \\
\hline Additional resection after iMRI (\%) & $48 / 181(26.5)$ & $38 / 121(31.4)$ & $8 / 50(16)$ & 0.411 \\
\hline New hypopituitarism after surgery (\%) & $44 / 149(29.5)$ & $32 / 104(30.8)$ & $9 / 38(23.7)$ & \\
\hline Boldface type indicates statistical significance. & & & &
\end{tabular}




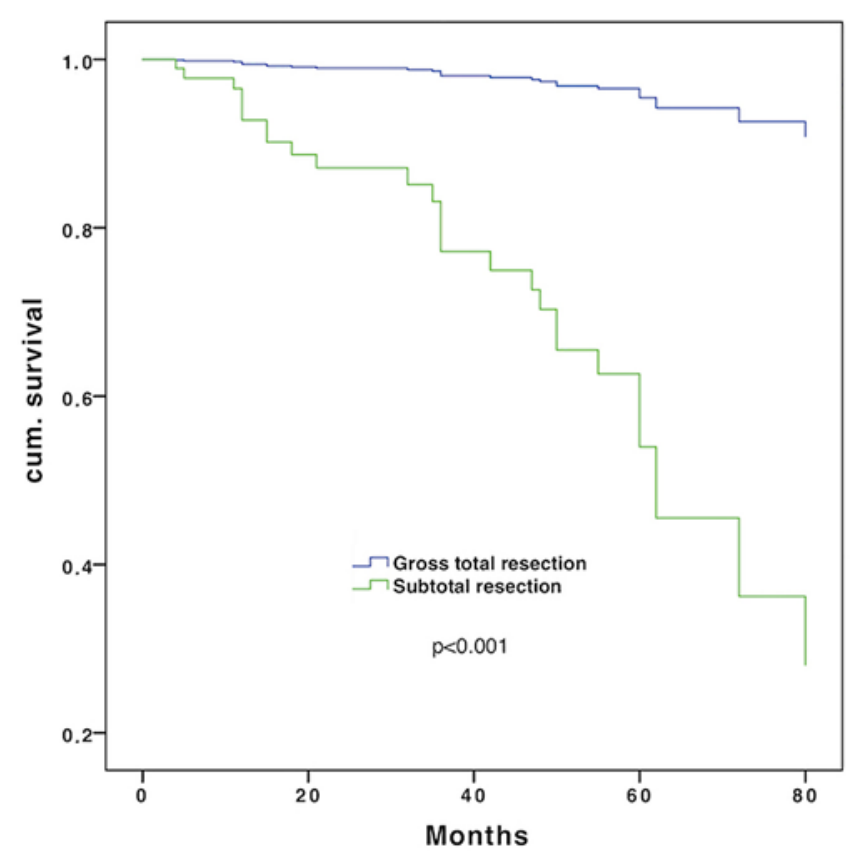

FIG. 1. Cox regression model of PFS and EOR. cum. = cumulative.

tumor volume relevant for adenoma recurrence. The calculated cutoff value was $0.008 \mathrm{~cm}^{3}$, and the results are depicted in Fig. 2.

\section{Endocrine Outcome}

New hypopituitarism was defined as the worsening of one or more pituitary axes after surgery. We found $28.2 \%$ new persistent endocrine deficits (Table 2). The endoscopic technique was significantly associated with fewer new persistent pituitary deficits. New permanent DI was diagnosed in $5.3 \%$ of cases (16 cases). The endoscopic approach was associated with a significantly lower rate of new permanent DI after surgery $(\mathrm{p}=0.027$; endoscopy, 2 cases; microscopy, 12 cases; endoscope-assisted, 2 cases). In the subgroup analysis of patients with Knosp grade $0-2$ adenomas, we found no significant difference in endocrine outcome between surgical techniques (Table 2). Complete biochemical remission in functional adenomas was reached in $58.1 \%$ of patients (25/43 cases). We found no significant association between additional adenoma resection after iMRI and new endocrine deficit $\left(\chi^{2}\right.$ $=0.734)$.

\section{Visual Function}

In 145 cases (48.3\%), visual deficits were documented prior to surgery. Of these, $78.6 \%$ (114 cases) improved after surgery. In only 3 cases $(1.0 \%)$ was new visual deterioration found after treatment. No significant difference was found between surgical techniques in regard to visual improvement after surgery $(\mathrm{p}=0.165)$.

\section{Complications}

Surgical revision was performed in $7.3 \%$ of cases (Table 6), most commonly because of a CSF fistula. In 2 cases, revision was needed due to postoperative hemorrhage, in 1 case because of new hydrocephalus, and in 1 patient because of visual deterioration following intracranial dislocation of a fat graft causing compression of the optic nerve. One patient needed local revision because of prolonged epistaxis after surgery. We found no significant difference in postoperative complications between surgical techniques $(p=0.709)$. Simultaneously, no significant association was seen between surgical technique and CSF fistula ( $\mathrm{p}=0.824)$. Meningitis was a rare complication (2.3\%). Surgical mortality in our cohort reached $1 \%$ (3 cases). In all cases it was related to vascular injury.

\section{Discussion \\ Extent of Resection}

Pituitary adenomas are benign lesions that can often be resected in a straightforward fashion. Yet, accidentally left residual tumor can recur and invade parasellar structures, making resection at a later stage more challenging or even not feasible. ${ }^{1}$ iMRI may increase GTR rates and improve overall outcome, as suggested by other groups examining smaller patient samples. ${ }^{4,15,20}$ The aim of our study was to analyze our long-term experience with both endoscopic and microsurgical approaches in the iMRI environment in a large sample covering the whole variety of pituitary cases. We conducted a retrospective analysis of our institutional experience with iMRI-assisted transsphenoidal resection of pituitary adenomas over 10 years. We performed a multivariable Cox regression analysis of PFS. In

TABLE 4. Univariable and multivariable Cox regression analyses of PFS

\begin{tabular}{|c|c|c|c|c|c|c|}
\hline \multirow[b]{2}{*}{ Variable } & \multicolumn{3}{|c|}{ Univariable Analysis } & \multicolumn{3}{|c|}{ Multivariable Analysis } \\
\hline & $p$ Value & $\mathrm{HR}$ & $95 \% \mathrm{Cl}$ & $\mathrm{p}$ Value & HR & $95 \% \mathrm{Cl}$ \\
\hline Age & 0.042 & 0.974 & $0.950-0.999$ & 0.100 & 0.978 & $0.952-1.004$ \\
\hline GTR & $<0.001$ & 0.086 & $0.030-0.249$ & $<0.001$ & 0.069 & $0.013-0.358$ \\
\hline Recurrent surgery & 0.253 & 0.629 & $0.284-1.393$ & & & \\
\hline Knosp grade & 0.003 & 3.310 & $1.506-7.278$ & 0.545 & 0.756 & $0.306-1.868$ \\
\hline Sex & 0.713 & 0.863 & $0.392-1.896$ & & & \\
\hline Initial tumor vol & 0.003 & 1.051 & $1.017-1.086$ & 0.668 & 1.009 & $0.970-1.049$ \\
\hline Adenoma subtype & 0.472 & & & & & \\
\hline
\end{tabular}

Boldface type indicates statistical significance. 
TABLE 5. Univariable and multivariable Cox regression analyses of PFS

\begin{tabular}{|c|c|c|c|c|c|c|}
\hline \multirow[b]{2}{*}{ Variable } & \multicolumn{3}{|c|}{ Univariable Analysis } & \multicolumn{3}{|c|}{ Multivariable Analysis } \\
\hline & $p$ Value & HR & $95 \% \mathrm{Cl}$ & $p$ Value & HR & $95 \% \mathrm{Cl}$ \\
\hline Age & 0.042 & 0.974 & $0.950-0.999$ & 0.029 & 0.970 & $0.944-0.997$ \\
\hline Postop tumor vol & $<0.001$ & 1.350 & $1.187-1.536$ & $<0.001$ & 1.417 & $1.197-1.676$ \\
\hline Recurrent surgery & 0.253 & 0.629 & $0.284-1.393$ & & & \\
\hline Knosp grade & 0.003 & 3.310 & $1.506-7.278$ & 0.021 & 0.345 & $0.140-0.849$ \\
\hline Sex & 0.713 & 0.863 & $0.392-1.896$ & & & \\
\hline Initial tumor vol & 0.003 & 1.051 & $1.017-1.086$ & 0.124 & 0.958 & $0.906-1.012$ \\
\hline Adenoma subtype & 0.472 & & & & & \\
\hline
\end{tabular}

Boldface type indicates statistical significance.

our model, age, residual tumor volume, and Knosp grade were confirmed as independent positive predictors of PFS. For residual tumor volume, we further calculated the ROC curve to identify a cutoff volume of residual tumor for which PFS starts to decline significantly. The extremely low value suggests that maximal resection, ideally GTR, should be the goal. Simultaneously, the volume of residual tumor is the only parameter in our model that can be influenced by the treatment. Since residual tumor can be reliably detected by iMRI, the surgeon has the opportunity to enhance the EOR. We can postulate that, by increasing the EOR and thereby reducing the volume of residual tumor, iMRI has a positive impact on PFS in pituitary adenoma patients.

The routine use of iMRI seems to lead to higher GTR rates and a significantly greater EOR based on our volumetric data. To the best of our knowledge, this is the larg-

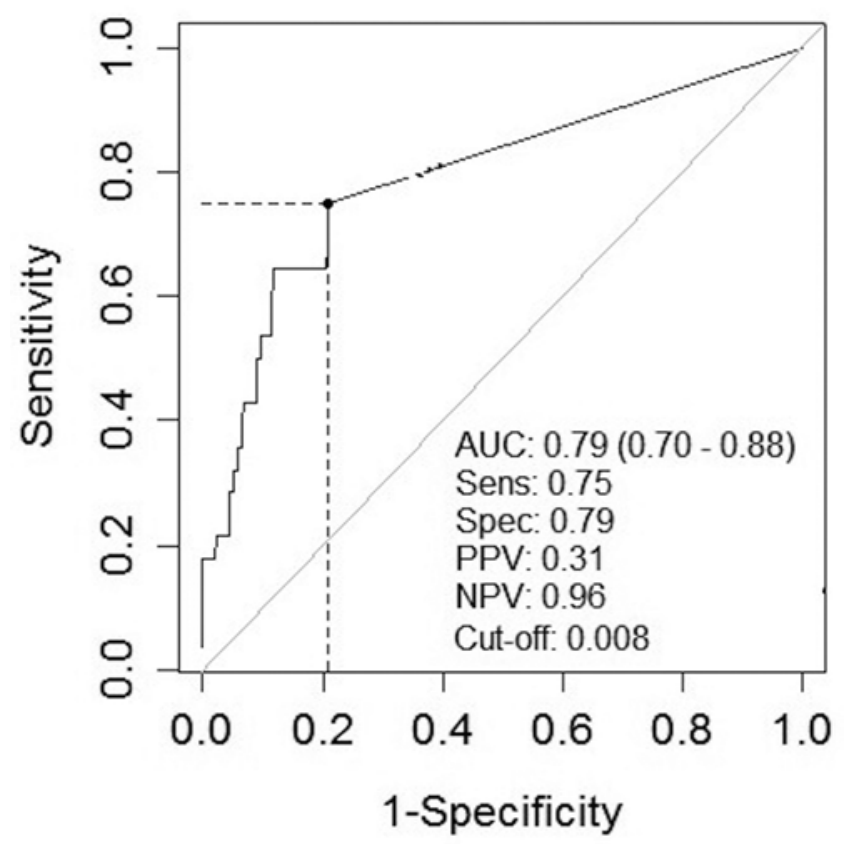

FIG. 2. ROC curve model of cutoff values for residual tumor volume relevant for adenoma recurrence. $\mathrm{AUC}=$ area under the curve; NPV = negative predictive value; $P P V=$ positive predictive value; Sens $=$ sensitivity; Spec = specificity. est cohort of patients who have undergone iMRI-assisted pituitary surgery so far. In a study based on the evaluation of 156 cases treated with the use of iMRI, Sylvester et al. reported that additional resection was performed in 35.9\% of cases and that EOR increased in $9.6 \%$ of cases. ${ }^{20}$ Our data from 300 cases are similar, confirming additional resection after iMRI in more than one-third of adenomas. Furthermore, we compared two common surgical techniques in the iMRI setting and found no significant difference between the two with regard to EOR among the whole cohort. This finding is also supported by the recently published prospective TRANSSPHER (Transsphenoidal Extent of Resection) study, which found no difference in GTR and EOR between microsurgical and endoscopic techniques in a similarly large cohort, although no iMRI was used in that study. ${ }^{11}$ In our study, some bias may have been introduced by the larger tumor volumes and higher Knosp grades in the endoscopic cohort since both have been proposed as important factors influencing EOR. ${ }^{6,9,22}$

However, our subgroup analysis of adenomas without invasion into the cavernous sinus, classified as Knosp grades $0-2$, was associated with a significantly greater EOR with a higher proportion of GTRs when the endoscopic technique was used. Simultaneously, additional resection was significantly more common with the microsurgical technique. These data encourage the routine use of iMRI especially if the microsurgical technique is used. Nevertheless, in almost one-fifth of the patients with Knosp grade $0-2$ adenomas treated by the endoscopic technique, iMRI was beneficial and resulted in additional resection. Even if an initial learning curve with endoscopy might have influenced our data, the routine use of iMRI seems to be justifiable in pituitary surgery independent of surgical technique. Furthermore, the low rate of postoperative meningitis, which is comparable with that in the published literature, underlines the fact that the longer operation time with iMRI does not result in a higher rate of infections or further complications. ${ }^{12,14}$ Similarly, in a previous study, we showed that a longer surgical time due to iMRI does not lead to higher complication rates. ${ }^{5}$ Even considering more aggressive adenomas invading the cavernous sinus, iMRI still increases EOR, as previously reported by our group. ${ }^{8}$ In the review by Singh et al. comparing the endoscopic and microsurgical techniques, they concluded that the two techniques are equal for smaller tumors but that endoscopy may be advantageous for larger 
TABLE 6. Complications

\begin{tabular}{lcccc}
\hline \multicolumn{1}{c}{ Complication } & $\begin{array}{c}\text { Total } \\
(\mathrm{n}=300)\end{array}$ & $\begin{array}{c}\text { Microsurgery Cohort } \\
(\mathrm{n}=187)\end{array}$ & $\begin{array}{c}\text { Endoscopy Cohort } \\
(\mathrm{n}=100)\end{array}$ & Microsurgical Endoscope-Assisted Cohort \\
\hline Surgical revision & $22(7.3)$ & $11(5.9)$ & $11(11)$ & - \\
\hline CSF fistula & $17(5.7)$ & $8(4.3)$ & $9(9)$ & - \\
\hline Meningitis & $7(2.3)$ & $5(2.7)$ & $2(2)$ & - \\
\hline Visual deterioration & $3(1.0)$ & $2(1.1)$ & $1(1)$ & - \\
\hline New permanent neurological deficit & $5(1.7)$ & $4(2.1)$ & $1(1)$ & - \\
\hline Epistaxis & $4(1.3)$ & $3(1.6)$ & $1(1)$ & - \\
\hline
\end{tabular}

Values are expressed as number (\%).

tumors..$^{18}$ If we consider the additional value of iMRI in our study, it may be especially beneficial for the microsurgical technique and may compensate for the limited field of view provided by the technique. Nevertheless, according to our data, iMRI seems to be advantageous even for the endoscopic technique mainly in cases of large and invasive adenomas. One of the advantages of iMRI is the possibility to reevaluate anatomical borders and to optimize the parasellar extension of the approach with regard to the internal carotid artery. Thus, iMRI may increase the safety of the transsphenoidal procedure as well as increase the EOR. In our experience, intraoperative imaging offers high image quality with very low rates of either artifacts or ambiguous findings.

\section{Progression-Free Survival}

GTR is the main surgical goal in many intracranial pathologies and was confirmed as an independent predictor of PFS in our multivariable Cox regression model. Postoperative scarring and loss of anatomical landmarks as well as the proximity of important vascular and neural structures in the parasellar region are the main arguments for avoiding recurrent surgery. ${ }^{16}$ Despite the benign nature of pituitary adenomas, they can recur and require further treatment. ${ }^{4}$ Therefore, GTR remains the chief aim of the primary resection. iMRI can support this goal by identifying obscure tumor remnants and thereby increasing the rate of GTR. Given the lack of a control group and the fact that iMRI was performed at the surgeon's discretion, we were not able to prove this hypothesis, but we feel confident that our data give us a strong indication in this direction.

We did not include surgical technique in our analysis of PFS since the endoscopic technique has been routinely used in our department since 2015. However, we are convinced that the combination of the endoscopic surgical technique and iMRI does have a synergistic effect and may offer some advantages if we consider the extended midline approaches.

As reported by Berkmann et al., the natural involution of pituitary adenomas after surgery surely influenced our data. ${ }^{3}$ We have confirmed this trend since even in patients without additional tumor resection, we found a significant tumor volume decrease during follow-up. On the other hand, our data advocate for the maximal possible resection since even small tumor remnants are independent negative prognostic factors for PFS.

\section{Visual and Endocrine Outcome}

Visual improvement is another main goal of pituitary surgery. ${ }^{7}$ Our data confirm a very high proportion of visual improvements achieved by transsphenoidal decompression of the optic nerve. ${ }^{12,14}$ Our results are congruent with those in the current literature. ${ }^{4,14}$ In terms of the endocrine outcome, the preservation of pituitary function and the attainment of biochemical remission are the main foci of pituitary surgery. Our cohort showed a relatively high frequency of pituitary disturbances. This may have been a consequence of the relatively high proportion of tumor recurrences as well as invasive adenomas. New hypopituitarism was classified as such even if only one axis was affected. Similarly, as published by Berkmann et al., we found no significant association between additional tumor resection and new endocrine deficit. ${ }^{2}$ Berkmann and colleagues showed a slightly higher number of new hypopituitarism cases compared to that in our study; ${ }^{4}$ however, they only used microsurgical or endoscope-assisted microsurgical approaches in their study. Laws et al. suggested that the endoscopic approach may be beneficial for endocrine outcome given better visualization and delineation of the pituitary gland. ${ }^{10}$ Similarly, we found significantly better endocrine outcomes using the endoscope in our initial experience. We can confirm this trend in the current study cohort since the endoscopic technique was significantly related to a better endocrine outcome. However, this effect seems to be less pronounced for pituitary adenomas without invasion into the cavernous sinus. The microsurgical view may be less accurate for localization of the pituitary stalk in complex cases and invasive adenomas. Here may lie another benefit of endoscopy. iMRI-assisted pituitary surgery may lead to more aggressive resection and potentially result in a higher rate of hypopituitarism. Our data cannot answer this question, and only direct comparison of iMRI with complete non-iMRI transsphenoidal surgery can resolve this question. We found only a nonsignificant association in the evaluation of persistent DI after surgery. On the contrary, as recently published by our group, iMRIassisted endoscopic resection of invasive Knosp grade 3-4 pituitary adenomas showed a significant advantage. ${ }^{8}$ This result supports previously mentioned advantages of the endoscopic approach.

\section{Study Limitations}

A retrospective design as well as the monocentric na- 
ture of our study could have biased our results. Furthermore, iMRI was performed at the surgeon's discretion, so it is not possible to exclude that the opportunity for additional resection after iMRI influenced the decision to scan and ultimately influenced the overall resection strategy. The data on intraoperative tumor volume do not allow any comparison with postoperative data on patients operated on without iMRI. Since all elective pituitary adenoma surgeries in our department are planned for iMRI-assisted tumor resection, we do not have any patient cohort operated on without iMRI with which to compare the presented data. If we consider the relatively large EOR provided by experienced hands with and without iMRI, it would be difficult to design a sufficiently powered prospective randomized study to address the question of the superiority of either method. Moreover, patients with incomplete endocrinological follow-up were excluded from the analysis of endocrine function. This is another source of bias that may have influenced our data. Furthermore, we do not compare EOR to results without iMRI. This fact is based on the clinical management of our department in which all elective pituitary adenoma surgeries are planned for iMRIassisted tumor resection. Moreover, the follow-up period is still relatively short with regard to this slowly growing lesion, and this fact could have biased our data.

New surgical techniques, such as dissection along the pseudocapsule, surely have an impact on EOR and could result in GTR even without the need for further visualization with iMRI. Unfortunately, we did not analyze this aspect in our results, and further studies should answer this question. ${ }^{21}$ However, from our point of view, iMRI is an additional safety loop especially in difficult situations such as scarring in recurrent surgery, lost anatomical borders or tumor growing beyond the lateral internal carotid artery, or rigid adenomas. In the case of successful pseudocapsular dissection, iMRI is probably mostly unnecessary, but this situation is rarely predictable, and iMRI may increase the safety and achievability of this goal. Even if iMRI influences a surgeon's decision-making, based on our experience, it is mostly beneficial with regard to EOR without additional complications.

\section{Conclusions}

In our study, additional tumor resection after iMRI reduced the residual volume of pituitary adenoma after both microsurgical and endoscopic transsphenoidal surgery. The reduction of tumor volume led to significantly prolonged PFS. EOR, age, and Knosp grade were independent predictors of PFS. According to our data, it is encouraging to strive for GTR whenever possible.

\section{References}

1. Almutairi RD, Muskens IS, Cote DJ, Dijkman MD, Kavouridis VK, Crocker E, et al: Gross total resection of pituitary adenomas after endoscopic vs. microscopic transsphenoidal surgery: a meta-analysis. Acta Neurochir (Wien) 160:10051021,2018

2. Berkmann S, Fandino J, Müller B, Remonda L, Landolt H: Intraoperative MRI and endocrinological outcome of transsphenoidal surgery for non-functioning pituitary adenoma. Acta Neurochir (Wien) 154:639-647, 2012
3. Berkmann S, Schlaffer S, Buchfelder M: Tumor shrinkage after transsphenoidal surgery for nonfunctioning pituitary adenoma. J Neurosurg 119:1447-1452, 2013

4. Berkmann S, Schlaffer S, Nimsky C, Fahlbusch R, Buchfelder M: Follow-up and long-term outcome of nonfunctioning pituitary adenoma operated by transsphenoidal surgery with intraoperative high-field magnetic resonance imaging. Acta Neurochir (Wien) 156:2233-2243, 2014

5. Coburger J, König R, Seitz K, Bäzner U, Wirtz CR, Hlavac M: Determining the utility of intraoperative magnetic resonance imaging for transsphenoidal surgery: a retrospective study. J Neurosurg 120:346-356, 2014

6. Dallapiazza R, Bond AE, Grober Y, Louis RG, Payne SC, Oldfield EH, et al: Retrospective analysis of a concurrent series of microscopic versus endoscopic transsphenoidal surgeries for Knosp Grades 0-2 nonfunctioning pituitary macroadenomas at a single institution. J Neurosurg 121:511-517, 2014

7. Frank G, Pasquini E, Farneti G, Mazzatenta D, Sciarretta V, Grasso V, et al: The endoscopic versus the traditional approach in pituitary surgery. Neuroendocrinology 83:240248, 2006

8. Hlaváč M, Knoll A, Etzrodt-Walter G, Sommer F, Scheithauer M, Coburger J, et al: Intraoperative MRI in transsphenoidal resection of invasive pituitary macroadenomas. Neurosurg Rev 42:737-743, 2019

9. Knosp E, Steiner E, Kitz K, Matula C: Pituitary adenomas with invasion of the cavernous sinus space: a magnetic resonance imaging classification compared with surgical findings. Neurosurgery 33:610-618, 1993

10. Laws ER Jr, Iuliano SL, Cote DJ, Woodmansee W, Hsu L, Cho $\mathrm{CH}$ : A benchmark for preservation of normal pituitary function after endoscopic transsphenoidal surgery for pituitary macroadenomas. World Neurosurg 91:371-375, 2016

11. Little AS, Kelly DF, White WL, Gardner PA, FernandezMiranda JC, Chicoine MR, et al: Results of a prospective multicenter controlled study comparing surgical outcomes of microscopic versus fully endoscopic transsphenoidal surgery for nonfunctioning pituitary adenomas: the Transsphenoidal Extent of Resection (TRANSSPHER) Study. J Neurosurg 132:1043-1053, 2020

12. Magro E, Graillon T, Lassave J, Castinetti F, Boissonneau S, Tabouret E, et al: Complications related to the endoscopic endonasal transsphenoidal approach for nonfunctioning pituitary macroadenomas in 300 consecutive patients. World Neurosurg 89:442-453, 2016

13. Micko ASG, Wöhrer A, Wolfsberger S, Knosp E: Invasion of the cavernous sinus space in pituitary adenomas: endoscopic verification and its correlation with an MRI-based classification. J Neurosurg 122:803-811, 2015

14. Mortini P, Losa M, Barzaghi R, Boari N, Giovanelli M: Results of transsphenoidal surgery in a large series of patients with pituitary adenoma. Neurosurgery 56:1222-1233, 2005

15. Pal'a A, Knoll A, Brand C, Etzrodt-Walter G, Coburger $\mathrm{J}$, Wirtz CR, et al: The value of intraoperative magnetic resonance imaging in endoscopic and microsurgical transsphenoidal pituitary adenoma resection. World Neurosurg 102:144-150, 2017

16. Pomeraniec IJ, Dallapiazza RF, Xu Z, Jane JA Jr, Sheehan JP: Early versus late Gamma Knife radiosurgery following transsphenoidal resection for nonfunctioning pituitary macroadenomas: a matched cohort study. J Neurosurg 125:202212, 2016

17. Schaberg MR, Anand VK, Schwartz TH, Cobb W: Microscopic versus endoscopic transnasal pituitary surgery. Curr Opin Otolaryngol Head Neck Surg 18:8-14, 2010

18. Singh H, Essayed WI, Cohen-Gadol A, Zada G, Schwartz TH: Resection of pituitary tumors: endoscopic versus microscopic. J Neurooncol 130:309-317, 2016 
19. Soneru CP, Riley CA, Hoffman K, Tabaee A, Schwartz TH: Intra-operative MRI vs endoscopy in achieving gross total resection of pituitary adenomas: a systematic review. Acta Neurochir (Wien) 161:1683-1698, 2019

20. Sylvester PT, Evans JA, Zipfel GJ, Chole RA, Uppaluri R, Haughey BH, et al: Combined high-field intraoperative magnetic resonance imaging and endoscopy increase extent of resection and progression-free survival for pituitary adenomas. Pituitary 18:72-85, 2015

21. Taylor DG, Jane JA, Oldfield EH: Resection of pituitary macroadenomas via the pseudocapsule along the posterior tumor margin: a cohort study and technical note. J Neurosurg 128:422-428, 2018

22. Zaidi HA, Awad AW, Bohl MA, Chapple K, Knecht L, Jahnke H, et al: Comparison of outcomes between a less experienced surgeon using a fully endoscopic technique and a very experienced surgeon using a microscopic transsphenoidal technique for pituitary adenoma. J Neurosurg 124:596-604, 2016

\section{Disclosures}

Dr. Coburger is a consultant for Brainlab AG.

\section{Author Contributions}

Conception and design: Pal'a, Hlaváč. Acquisition of data: Pal'a, Knoll, Braun, Etzrodt-Walter. Analysis and interpretation of data: Pal'a, Hlaváč, Knoll, Braun, Etzrodt-Walter, Coburger, Wirtz. Drafting the article: Pal'a, Hlaváč, Mayer. Critically revising the article: Knoll, Mayer, Braun, Karpel-Massler, Coburger, Wirtz. Approved the final version of the manuscript on behalf of all authors: Pal'a. Statistical analysis: Pal'a, Mayer.

\section{Correspondence}

Andrej Pal'a: University of Ulm, Günzburg, Germany. andrej. pala@gmail.com. 